Pacific Journal of Mathematics

TRACEABLE INTEGRAL KERNELS ON COUNTABLY
GENERATED MEASURE SPACES 


\section{TRACEABLE INTEGRAL KERNELS \\ ON COUNTABLY GENERATED MEASURE SPACES}

\section{CHRIS BRISLAWN}

Let $K$ be a trace class operator on $L^{2}(X, \mathscr{M}, \mu)$ with integral kernel $K(x, y) \in L^{2}(X \times X, \mu \times \mu)$. An averaging process is used to define $K$ on the diagonal in $X \times X$ so that the trace of $K$ is equal to the integral of $K(x, x)$, generalizing results known previously for continuous kernels. This formula is also shown to hold for positive-definite Hilbert-Schmidt operators, thus giving necessary and sufficient conditions for the traceability of positive integral kernels. These results make use of Doob's maximal theorem for martingales and generalize previous results obtained by the author using HardyLittlewood maximal theory when $X \subset \mathbf{R}^{n}$.

1. Introduction. Let $K$ be a compact operator on a Hilbert space, H. The positive operator $K^{*} K$ can be diagonalized by an orthonormal sequence $\left\{\psi_{i}\right\}_{i \in \mathbf{N}}$ of eigenvectors with corresponding eigenvalues $\mu_{i}>0$. Define $\lambda_{i}=\sqrt{\mu_{i}}$ and $\phi_{i}=\lambda_{i}^{-1} K \psi_{i}$. The numbers $\lambda_{i}$, the eigenvalues of $|K|$, are called the singular values of $K$, and the sequence $\left\{\phi_{i}\right\}_{i \in \mathbf{N}}$ is also orthonormal. $K$ is a Hilbert-Schmidt operator if the singular values are square-summable and a trace class operator if they are absolutely summable. Since $\operatorname{ker}(K)=\operatorname{ker}\left(K^{*} K\right)$, we have

$$
\operatorname{cl} \operatorname{span}\left\{\psi_{i}\right\}_{i \in \mathbf{N}}=\operatorname{ker}\left(K^{*} K\right)^{\perp}=\operatorname{ker}(K)^{\perp} .
$$

Let $\phi \otimes \bar{\psi}$ denote the rank 1 operator

$$
\phi \otimes \bar{\psi}: \theta \mapsto(\theta, \psi) \phi .
$$

By expanding $\theta \in \operatorname{ker}(K)^{\perp}$ in terms of the basis $\left\{\psi_{i}\right\}_{i \in \mathbf{N}}$, we see that

$$
K \theta=\sum_{i=1}^{\infty} \lambda_{i}\left(\theta, \psi_{i}\right) \phi_{i}
$$

Thus, $K$ has the canonical expansion

$$
K=\sum_{i=1}^{\infty} \lambda_{i} \phi_{i} \otimes \bar{\psi}_{i},
$$

norm-convergent in $\mathscr{L}(H)$. 
We shall be interested in operators on the Hilbert spaces $\mathrm{L}^{2}(X, \mu)$. In this setting, every Hilbert-Schmidt operator, $K$, on $\mathrm{L}^{2}(X)$ is given by an integral kernel $K(x, y) \in \mathrm{L}^{2}(X \times X, \mu \times \mu)$ :

$$
K f(x)=\int_{X} K(x, y) f(y) d \mu(y) .
$$

The canonical expansion (1.2) corresponds to an orthonormal expansion of the kernel,

$$
K(x, y)=\sum \lambda_{i} \phi_{i}(x) \overline{\psi_{i}(y)}
$$

convergent in $\mathrm{L}^{2}(X \times X)$.

In a previous paper [1], it was shown that the ubiquitous trace formula

$$
\operatorname{tr} K=\int_{X} K(x, x) d \mu(x)
$$

can be extended for arbitrary trace class operators with no continuity assumptions on the kernel function when $\mu$ is Lebesgue measure on a set $X \subset \mathbf{R}^{n}$. Specifically, the diagonal values are defined by averaging the kernel over cubes centered on the diagonal in $\mathbf{R}^{2 n}$ and letting the radius of the cubes go to zero. That this limit exists almost everywhere on the diagonal and that the integral of the resulting function furnishes the trace of $K$ follow from the boundedness on $\mathrm{L}^{2}\left(\mathbf{R}^{n}\right)$ of the Hardy-Littlewood maximal operator. These results establish a certain degree of function-theoretic smoothness for traceable integral kernels and strengthen the analogy between noncommutative integration in $\mathscr{L}(\mathbf{H})$ and classical integration theory. They also provide completely general necessary and sufficient conditions for traceability of positive-definite integral operators.

The present paper extends these results from $\mathbf{R}^{n}$ to a general measure-theoretic setting. We shall obtain all the results in [1] with $\mathbf{R}^{n}$ replaced by a countably generated measure space, $(X, \mathscr{M})$, and Lebesgue measure by a $\sigma$-finite measure on $\mathscr{M}$. The canonical expansion (1.2) has been introduced to accommodate an eventual extension of the results of $\S 3$ to include nuclear operators on the Banach spaces $L^{p}(X)$. The diagonal values of an arbitrary trace class kernel, $K$, will be defined by an averaging process, with pointwise values denoted by $\widetilde{K}(x, x)$. We will then prove the analogue of $(1.5)$ :

$$
\operatorname{tr} K=\int \widetilde{K}(x, x) d \mu(x) .
$$


Conversely, we get the following generalization of Mercer's theorem: if $P \geq 0$ and $\widetilde{P}(x, x)$ exists for almost every $x$ with

$$
\int \widetilde{P}(x, x) d \mu(x)<\infty,
$$

then $P$ is traceable. Traceability also follows if the integrand in (1.6) is replaced by the corresponding maximal function, $\operatorname{MP}(x, x)$, generalizing a result of $\mathrm{J}$. Weidmann.

The key device in these proofs, a result from probability theory that replaces the Hardy-Littlewood theorem, is Doob's maximal theorem for martingales. The method of proof employed in [1] will be reformulated in a purely measure-theoretic way that does not depend on the geometric structure of Euclidean space. As an immediate corollary, we shall obtain a new proof of a result of $\mathrm{M}$. Duflo ([3], Théorème V.3.1.1): if $K(x, y)$ is a continuous trace class kernel on a $\mathrm{LCH}$ space then (1.5) holds. Our approach avoids the "analytic" hypotheses behind Duflo's proof; specifically, that $X$ be locally compact and $\sigma$-compact and that $\mu$ be a Radon measure. We shall only require that the topology on $X$ be countably generated and that $\mu$ be $\sigma$-finite on Borel sets.

Acknowledgments. The author wishes to thank Arlan Ramsay and Larry Baggett for much constructive advice, and Daniel Stroock for introducing the author to martingale theory.

2. Martingales. Let $(X, \mathscr{M}, \mu)$ be a $\sigma$-finite measure space and $\left\{\mathscr{S}_{n}\right\}_{n \in \mathbf{N}}$ a sequence of sub- $\sigma$-algebras increasing to $\mathscr{M}$ :

$$
\mathscr{S}_{n} \subset \mathscr{S}_{n+1} \text { and } \mathscr{M}=\bigvee_{n \in \mathbf{N}} \mathscr{S}_{n} \text {. }
$$

Assume that $\mu$ is $\sigma$-finite on each $\mathscr{S}_{n}$ so that, for $f \in \mathrm{L}^{p}(X)$, the conditional expectations $\mathrm{E}\left(f \mid \mathscr{S}_{n}\right)$ exist. A sequence $\left\{f_{n}\right\}_{n \in \mathbf{N}}$ of functions on $X$ is a martingale if each $f_{n}$ is $\mathscr{S}_{n}$-measurable and

$$
\mathrm{E}\left(f_{n} \mid \mathscr{S}_{j}\right)=f_{j}, \quad \text { whenever } j<n \text {. }
$$

Of particular interest to us are martingales generated by a single $\mathscr{M}$ measurable function, $f$, with $f_{n}=\mathrm{E}\left(f \mid \mathscr{S}_{n}\right)$. The maximal function of such a martingale is

$$
M f(x)=\sup _{n \in \mathbf{N}} \mathrm{E}\left(|f| \mid \mathscr{S}_{n}\right)(x) .
$$

There is a boundedness theorem for the martingale maximal operator analogous to the Hardy-Littlewood maximal theorem. The original 
reference is Doob ([2], Theorem VII.3.2); for treatments geared more for our purposes, see Stein ([8], Theorem IV.1.6), or Edwards and Gaudry ([4], Theorem 5.2.7).

Theorem 2.1. (Doob's Martingale Maximal Theorem.) The maximal operator, $M$, for martingales is weak-type $(1,1)$ on $\mathrm{L}^{1}(X)$ and bounded on $\mathrm{L}^{p}(X)$ for $1<p \leq \infty$.

The fact we shall need in $\S 3$ is that the martingale maximal operator is bounded on $\mathrm{L}^{2}(X)$ :

$$
\|M f\|_{2} \leq C_{2}\|f\|_{2} \text { for all } f \in \mathrm{L}^{2}(X) .
$$

The analogue of Lebesgue's differentiation theorem is:

Theorem 2.2. (Doob's Martingale Convergence Theorem.) For each $f \in \mathrm{L}^{p}(X), 1 \leq p \leq \infty$, the sequence $\mathrm{E}\left(f \mid \mathscr{S}_{n}\right)$ converges to $f$ a.e. $[\mu]$.

We shall construct sub- $\sigma$-algebras for which the conditional expectations $E\left(f \mid \mathscr{S}_{n}\right)$ are given by averaging $f$ over sets in $\mathscr{S}_{n}$. For two partitions $\mathscr{P}_{i}, \mathscr{P}_{j}$ of $X$, the notation $\mathscr{P}_{i} \prec \mathscr{P}_{j}$ will mean that $\mathscr{P}_{i}$ is a refinement of $\mathscr{P}_{j}$. Suppose $\left\{\mathscr{P}_{n}\right\}_{n \in \mathbf{N}}$ is a sequence of partitions of $X$ into sets of finite measure such that $\mathscr{P}_{n+1} \prec \mathscr{P}_{n}$. Let $\mathscr{S}_{n}=\sigma\left(\mathscr{P}_{n}\right)$, and suppose further that $\mathscr{M}=\bigvee_{n} \mathscr{S}_{n}$. For each $x \in X$ and each $n \in \mathbf{N}$ there is a unique set $C_{n}(x) \in \mathscr{P}_{n}$ containing $x$. The conditional expectation of $f$ with respect to $\mathscr{S}_{n}$ is

$$
\mathrm{E}\left(f \mid \mathscr{S}_{n}\right)(x)=\frac{1}{\mu\left(C_{n}(x)\right)} \int_{C_{n}(x)} f d \mu,
$$

whenever $\mu\left(C_{n}(x)\right) \neq 0$. Assume that each $\mathscr{P}_{n}$ is a countable partition of $X$ and let $\mathcal{N}$ be the set of all $x$ for which $\mu\left(C_{n}(x)\right)=0$ for some $n$ (and thus for all $m>n$, since $\left.C_{n+1}(x) \subset C_{n}(x)\right)$. Then $\mu(\mathscr{N})=0$, and for all $x \in \mathscr{N}^{c}=X \backslash \mathscr{N}$ the formula (2.3) holds.

With these hypotheses we can define an averaging process analogous to the Hardy-Littlewood process:

$$
\begin{aligned}
A_{n} f(x) & =\mathrm{E}\left(f \mid \mathscr{S}_{n}\right)(x) \\
& =\frac{1}{\mu\left(C_{n}(x)\right)} \int_{C_{n}(x)} f d \mu, \quad \text { for } x \in \mathscr{N}^{c} .
\end{aligned}
$$

It follows that the operators $A_{n}$ convert $\mathrm{L}^{p}$ convergence into pointwise convergence: if $\sum f_{i}$ converges to $f$ in $\mathrm{L}^{p}(X)$ then at each 
$x \in \mathscr{N}^{c}$

$$
\sum A_{n} f_{i}(x)=A_{n} f(x) .
$$

On $X \times X$ we have induced partitions $\mathscr{P}_{n} \times \mathscr{P}_{n}$, with each point $(x, y) \in C_{n}(x) \times C_{n}(y)$ and $\mathscr{P}_{n+1} \times \mathscr{P}_{n+1} \prec \mathscr{P}_{n} \times \mathscr{P}_{n}$. The set $\mathscr{N}^{c} \times \mathscr{N}^{c}$ is conull with respect to product measure, and note that almost all of the diagonal lies in $\mathscr{N}^{c} \times \mathscr{N}^{c}$ :

$$
(x, x) \in \mathscr{N}^{c} \times \mathscr{N}^{c} \text { a.e. }[\mu] .
$$

Let $A_{n}^{(2)}$ denote the averaging operators on $X \times X$. When $(x, y) \in$ $\mathscr{N}^{c} \times \mathscr{N}^{c}$,

(2.7) $A_{n}^{(2)} f(x, y)$

$$
=\frac{1}{\mu\left(C_{n}(x)\right) \mu\left(C_{n}(y)\right)} \int_{C_{n}(x)} \int_{C_{n}(y)} f(s, t) d \mu(t) d \mu(s) .
$$

Applying (2.7) to elementary tensors,

$$
A_{n}^{(2)}(\phi \otimes \psi)(x, y)=A_{n} \phi(x) A_{n} \psi(y),
$$

for all $(x, y) \in \mathscr{N}^{c} \times \mathscr{N}^{c}$. Consequently,

LEMMA 2.3. The martingale maximal operator is submultiplicative and subadditive: if $(x, y) \in \mathscr{N}^{c} \times \mathscr{N}^{c}$ then

$$
M^{(2)}(\phi \otimes \psi)(x, y) \leq M \phi(x) M \psi(y)
$$

and

$$
M(\phi+\psi)(x) \leq M \phi(x)+M \psi(x) .
$$

Now let $\mathscr{U}=\left\{U_{n}\right\}_{n \in \mathbf{N}}$ be a countable base for $\mathscr{M}$, and use it to define a sequence of partitions on $X$. For $\mathscr{P}_{0}$ take a countable partition of $X$ into sets of finite measure, and define $\mathscr{P}_{n}$ inductively:

$$
\mathscr{P}_{n}=\left\{U_{n}, U_{n}^{c}\right\} \cap \mathscr{P}_{n-1}, \quad n \in \mathbf{N} .
$$

The $\cap$ in (2.9) means "all pairwise intersections." This is an increasing sequence of partitions, $\mathscr{P}_{n+1} \prec \mathscr{P}_{n}$, so $\mathscr{P}_{n}=\sigma\left(\mathscr{P}_{n}\right)$ is an increasing sequence of sub- $\sigma$-algebras of $\mathscr{M}$, and $\mathscr{M}=\bigvee_{n} \mathscr{S}_{n}$. For $f \in \mathrm{L}^{p}(X)$ define

$$
\tilde{f}(x)=\lim _{n \rightarrow \infty} A_{n} f(x)
$$

Then

$$
\tilde{f}(x)=f(x) \text { a.e. }[\mu]
$$


by Theorem 2.2. Call $x \in \mathscr{N}^{c}$ a regular point for $f$ if the limit (2.10) exists. Note that the set $Y_{f}$ of regular points for $f$ is conull in $X$. $Y_{f}$ should be thought of as the "Lebesgue set" of $f$ with respect to the measure $\mu$.

If $X$ is a second-countable topological space we shall let $\mathscr{U}$ be a countable base for the topology on $X$. The $\sigma$-algebras $\sigma\left(\mathscr{P}_{n}\right)$ will then converge to the Borel $\sigma$-algebra. Because the averaging process for this martingale is based on the topology of $X$, we have the following result:

THEOREM 2.4. Let $\mu$ be a $\sigma$-finite Borel measure on a secondcountable space, $X$. If $f \in \mathrm{L}^{p}(X, \mu), 1 \leq p \leq \infty$, then each point of continuity $x \in \mathscr{N}^{c}$ is a regular point for $f$, with $\tilde{f}(x)=f(x)$.

The same result holds in $X \times X$ with regard to the process (2.7). Because almost all of the diagonal lies in $\mathscr{N}^{c} \times \mathscr{N}^{c}$, if $f(x, y)$ is continuous almost everywhere along the diagonal then

$$
A_{n}^{(2)} f(x, x) \rightarrow f(x, x) \text { a.e. }[\mu],
$$

even though the diagonal is null with respect to product measure. Without any continuity hypotheses, we only have almost everywhere existence with respect to product measure:

$$
\tilde{f}(x, y)=\lim _{n \rightarrow \infty} A_{n}^{(2)} f(x, y) \text { a.e. }[\mu \times \mu] .
$$

Of course, if $\mu$ is a Borel measure on $\mathbf{R}^{m}$ then the construction (2.9) is unnecessary and one can define the partitions $\mathscr{P}_{n}$ by dyadic decompositions of $\mathbf{R}^{m}$ into half-open cubes:

$$
\left[j 2^{-n},(j+1) 2^{-n}\right) \times \cdots \times\left[k 2^{-n},(k+1) 2^{-n}\right) .
$$

These partitions satisfy $\mathscr{P}_{n+1} \prec \mathscr{P}_{n}$ and generate the Borel $\sigma$-algebra, so all of the above results apply, including Theorem 2.4. Note that the averaging process defined by these partitions satisfies Theorems 2.1 and 2.2 even though the Hardy-Littlewood maximal theorem may fail for averaging over balls with respect to the measure $\mu$. For certain $\mu$, however, one can smooth by averaging over balls with respect to Lebesgue measure; Muckenhoupt [5] has classified the weight functions $w(x)$ (the " $A_{p}$ weights") for which the ordinary HardyLittlewood maximal operator is bounded on the weighted space $\mathrm{L}^{2}\left(\mathbf{R}^{m}, w(x) d x\right)$. For more on this and related results see Torchinsky [9]. 
3. The trace of an integral kernel. Let $(X, \mathscr{M}, \mu)$ be a countably generated, $\sigma$-finite measure space and suppose that $K$ is a trace class operator on $\mathrm{L}^{2}(X, \mu)$ with kernel $K(x, y)$ in $\mathrm{L}^{2}(X \times X, \mu \times \mu)$. Consider an expansion of the form (1.4):

$$
K(x, y)=\sum \lambda_{i} \phi_{i}(x) \overline{\psi_{i}(y)} .
$$

We will say that $x \in \mathscr{N}^{c}$ is a regular point of the expansion (3.1) if, for all $i \in \mathbf{N}$, the limits

$$
\lim _{n \rightarrow \infty} A_{n} \phi_{i}(x)=\tilde{\phi}_{i}(x) \text { and } \lim _{n \rightarrow \infty} A_{n} \psi_{i}(x)=\tilde{\psi}_{i}(x)
$$

exist at $x$. That is, the set $Y_{\Sigma}$ of regular points for (3.1) is

$$
Y_{\Sigma}=\bigcap_{i \in \mathbf{N}}\left(Y_{\phi_{i}} \cap Y_{\psi_{\imath}}\right)
$$

Thus, almost every point in $X$ is a regular point by Theorem 2.2. From now on we will drop the tildes and consider $\phi_{i}$ and $\psi_{i}$ to be defined pointwise by (3.2) at each $x \in Y_{\Sigma}$. By (2.5) and (2.8) we have

$$
A_{n}^{(2)} K(x, y)=\sum \lambda_{i} A_{n} \phi_{i}(x) A_{n} \overline{\psi_{i}}(y)
$$

at every point $(x, y) \in \mathscr{N}^{c} \times \mathscr{N}^{c} . \widetilde{K}(x, y)$ is defined as above:

$$
\widetilde{K}(x, y)=\lim _{n \rightarrow \infty} A_{n}^{(2)} K(x, y),
$$

which exists pointwise a.e. $[\mu \times \mu]$.

We are now ready to state and prove the following generalization of Duflo's theorem:

Theorem 3.1. Let $K$ be a trace class operator on $\mathrm{L}^{2}(X, \mu)$; then the canonical expansion (3.1) for $K(x, y)$ converges absolutely a.e. $[\mu \times \mu]$ and a.e. $[\mu]$ on the diagonal, $M^{(2)} K(x, x) \in \mathrm{L}^{1}(X), \widetilde{K}(x, x)$ exists a.e. $[\mu]$, and

$$
\operatorname{tr} K=\int \widetilde{K}(x, x) d \mu(x)
$$

Proof. We have a canonical expansion for $K$ of the form (3.1),

$$
K(x, y)=\sum \lambda_{i} \phi_{i}(x) \overline{\psi_{i}(y)},
$$

with $\lambda_{i}>0$ and $\sum \lambda_{i}<\infty$. Since $K$ is traceable,

$$
\int \sum \lambda_{i}\left|\phi_{i}(x)\right|^{2}=\int \sum \lambda_{i}\left|\psi_{i}(x)\right|^{2}=\sum \lambda_{i}<\infty
$$


so the series $\sum \lambda_{i}\left|\phi_{i}(x)\right|^{2}$ and $\sum \lambda_{i}\left|\psi_{i}(x)\right|^{2}$ are finite almost everywhere. The inequality

$$
\left[\sum\left|\lambda_{i} \phi_{i}(x) \overline{\psi_{i}(y)}\right|\right]^{2} \leq \sum \lambda_{i}\left|\phi_{i}(x)\right|^{2} \sum \lambda_{i}\left|\psi_{i}(y)\right|^{2}
$$

shows that the canonical expansion converges absolutely a.e. $[\mu \times \mu]$ to $K(x, y)$ and that $\sum \lambda_{i} \phi_{i}(x) \overline{\psi_{i}(x)}$ converges absolutely a.e. $[\mu]$.

Using the results of $\S 2$, we have the following calculation:

$$
\begin{aligned}
\int M^{(2)} K(x, x) d x & \leq \int \sum \lambda_{i} M \phi_{i}(x) M \overline{\psi_{i}}(x) d \mu(x) \\
& \leq \sum \lambda_{i}\left\|M \phi_{i}\right\|_{2}\left\|M \overline{\psi_{i}}\right\|_{2} \\
& \leq C_{2}^{2} \sum \lambda_{i}\left\|\phi_{i}\right\|_{2}\left\|\overline{\psi_{i}}\right\|_{2} \\
& <\infty
\end{aligned}
$$

This proves the second assertion in the theorem and shows the convergence of the nonnegative series

$$
\sum \lambda_{i} M \phi_{i}(x) M \overline{\psi_{i}}(x)<\infty \text { a.e. }[\mu] \text {. }
$$

Choose a conull set $Y \subset Y_{\Sigma}$ of regular points for the canonical expansion so that for all $x \in Y$ each of the following three series is finite:

$$
\sum \lambda_{i}\left|\phi_{i}(x)\right|^{2}, \sum \lambda_{i}\left|\psi_{i}(x)\right|^{2}, \sum \lambda_{i} M \phi_{i}(x) M \overline{\psi_{i}}(x)
$$

Since $Y$ consists of regular points,

$$
\lim _{n \rightarrow \infty} A_{n} \phi_{i}(x)=\phi_{i}(x) \text { and } \lim _{n \rightarrow \infty} A_{n} \psi_{i}(x)=\psi_{i}(x),
$$

for all $x \in Y$ and all $i \in \mathbf{N}$. Now,

$$
\left|A_{n} \phi_{i}(x) A_{n} \overline{\psi_{i}}(x)\right| \leq M \phi_{i}(x) M \overline{\psi_{i}}(x)
$$

for $x \in Y$ so the series

$$
\sum \lambda_{i} A_{n} \phi_{i}(x) A_{n} \overline{\psi_{i}}(x)
$$

converges absolutely for each $x \in Y$. From (3.3) we know that

$$
A_{n}^{(2)} K(x, x)=\sum \lambda_{i} A_{n} \phi_{i}(x) A_{n} \overline{\psi_{i}}(x)
$$

so, letting $n \rightarrow \infty$, the dominated convergence theorem on $\mathbf{N}$ yields

$$
\begin{aligned}
\widetilde{K}(x, x) & =\lim _{n \rightarrow \infty} A_{n}^{(2)} K(x, x) \\
& =\sum \lambda_{i} \lim _{n \rightarrow \infty} A_{n} \phi_{i}(x) A_{n} \overline{\psi_{i}}(x) \\
& =\sum \lambda_{i} \phi_{i}(x) \overline{\psi_{i}(x)},
\end{aligned}
$$


for each $x \in Y$. Note that this series converges absolutely in $\mathrm{L}^{1}(X)$. Using (1.1), we compute the trace:

$$
\begin{aligned}
\operatorname{tr} K & =\sum\left(K \psi_{i}, \psi_{i}\right) \\
& =\sum \int\left(\lambda_{i} \phi_{i}(x) \overline{\psi_{i}(x)} d \mu(x)\right. \\
& =\int \sum \lambda_{i} \phi_{i}(x) \overline{\psi_{i}(x)} d \mu(x) \\
& =\int \widetilde{K}(x, x) d \mu(x) .
\end{aligned}
$$

In general, the function $\widetilde{K}(x, x)$ cannot be computed effectively because of the abstract nature of the averaging process defined in $\S 2$. The existence and integrability of $\widetilde{K}(x, x)$ is a completely general necessary condition for traceability, but Theorem 3.1 is most effective for actually computing the trace when $K(x, y)$ is continuous. Theorem 2.4 implies

Corollary 3.2. Let $\mu$ be a $\sigma$-finite Borel measure on a secondcountable space, $X$, and let $K$ be a trace class operator on $\mathrm{L}^{2}(X, \mu)$. If the kernel $K(x, y)$ is continuous at $(x, x)$ for almost every $x$ then

$$
\operatorname{tr} K=\int K(x, x) d \mu(x) \text {. }
$$

Now consider the trace formula given by factoring a trace class operator as a product of Hilbert-Schmidt operators: if $K=L J$, where $L, J$ are Hilbert-Schmidt operators, then

$$
\operatorname{tr} K=\int L * J(x, x) d \mu(x) .
$$

Although the integrand in (3.5),

$$
L * J(x, x)=\int L(x, z) J(z, x) d \mu(z),
$$

is a well-defined element of $\mathrm{L}^{1}(X)$, it is not clear that this function is independent of the particular factors $L$ and $J$, nor is it related in an obvious manner to the diagonal values of the kernel $K(x, y)$. As in [1], we can prove that, for any factorization $K=L J$, the function $L * J(x, x)$ coincides almost everywhere with $\widetilde{K}(x, x)$, so (3.5) agrees with the trace formula in Theorem 3.1. 
THEOREM 3.3. If $K=L J$ is a factorization of the trace class operator $K$ into a product of Hilbert-Schmidt operators, then

$$
\widetilde{K}(x, x)=L * J(x, x) \text { a.e. }[\mu] .
$$

The proof is identical to the proof of Theorem 3.5 in [1], using the averaging process constructed in $\S 2$ in lieu of the Hardy-Littlewood process.

4. Traceability of positive integral operators. Our final goal is to prove a version of Mercer's theorem (see [7] or [10]) that does not require continuity of the kernel. Along the way, we shall also generalize a theorem of Weidmann ([11], Satz 1$)$ and obtain its converse immediately from Theorem 3.1. This will give us two integral conditions, each of which is both necessary and sufficient for traceability of an arbitrary positive-definite kernel. We begin with a very easy lemma:

LemMa 4.1. Suppose that

$$
P=\sum_{i=1}^{\infty} \lambda_{i} \phi_{i} \otimes \overline{\phi_{i}}, \quad \lambda_{i}>0,
$$

is the eigenfunction expansion of a positive compact operator on $\mathrm{L}^{2}(X)$. If $x$ is a regular point for this expansion, then

$$
\sum \lambda_{i}\left|\phi_{i}(x)\right|^{2} \leq \liminf _{n \rightarrow \infty} \sum \lambda_{i}\left|A_{n} \phi_{i}(x)\right|^{2} .
$$

Proof. Since $x$ is a regular point, $A_{n} \phi_{i}(x) \rightarrow \phi_{i}(x)$ for all $i \in \mathbf{N}$, so (4.2) is just Fatou's lemma on $\mathbf{N}$.

Now for Weidmann's theorem and its converse in a more general setting than $\mathbf{R}^{n}$ :

THEOREM 4.2. Let $M P(x, y)$ be the maximal function of a positive Hilbert-Schmidt kernel. Then $P$ is traceable if and only if

$$
\int M P(x, x) d \mu(x)<\infty \text {. }
$$

Proof. As in $\S 3$,

$$
\sum \lambda_{i}\left|A_{n} \phi_{i}(x)\right|^{2}=A_{n}^{(2)} P(x, x)
$$

at each regular point, $x$. Apply Lemma 4.1

$$
\begin{aligned}
\sum \lambda_{i}\left|\phi_{i}(x)\right|^{2} & \leq \liminf _{n \rightarrow \infty} \sum \lambda_{i}\left|A_{n} \phi_{i}(x)\right|^{2} \\
& \leq M P(x, x)
\end{aligned}
$$


and integrate:

$$
\begin{aligned}
\operatorname{tr} P & =\sum \lambda_{i} \\
& =\int \sum \lambda_{i}\left|\phi_{i}(x)\right|^{2} d \mu(x) \leq \int M P(x, x) d \mu(x) .
\end{aligned}
$$

Thus, $P$ is traceable if $M P(x, x) \in L^{1}(X)$. The converse follows by Theorem 3.1.

Next, we obtain a generalization of Mercer's theorem with no compactness or continuity hypotheses:

THEOREM 4.3. Suppose that $P$ is a positive Hilbert-Schmidt operator and that $\widetilde{P}(x, x)$ exists a.e. $[\mu]$. Note that we allow the extended real value $\widetilde{P}(x, x)=+\infty$. If $\widetilde{P}(x, x)<\infty$ a.e. [ $\mu$ ] then expansion (4.1) converges pointwise:

$$
\widetilde{P}(x, y)=\sum \lambda_{i} \phi_{i}(x) \overline{\phi_{i}(y)} \text { a.e. }[\mu \times \mu] .
$$

Moreover,

$$
\operatorname{tr} P=\int \widetilde{P}(x, x) d x .
$$

Thus, $P$ is traceable if and only if $\widetilde{P}(x, x)$ exists for almost every $x$ and the integral (4.5) is finite.

Proof. First note that $\widetilde{P}(x, x) \geq 0$ a.e. $[\mu]$, by (4.3). For all regular points $x, y$ at which both $\widetilde{P}(x, x)$ and $\widetilde{P}(y, y)$ are finite, Lemma 4.1 implies

$$
\begin{aligned}
{\left[\sum\left|\lambda_{i} \phi_{i}(x) \overline{\phi_{i}(y)}\right|\right]^{2} } & \leq \sum \lambda_{i}\left|\phi_{i}(x)\right|^{2} \sum \lambda_{i}\left|\phi_{i}(y)\right|^{2} \\
& \leq \widetilde{P}(x, x) \widetilde{P}(y, y)<\infty
\end{aligned}
$$

so (4.4) converges absolutely a.e. $[\mu \times \mu]$, and the pointwise limit necessarily agrees with $P(x, y)$ almost everywhere. Lemma 4.1 and (4.3) also imply

$$
\begin{aligned}
\operatorname{tr} P & =\int \sum \lambda_{i}\left|\phi_{i}(x)\right|^{2} d \mu(x) \\
& \leq \int \liminf _{n \rightarrow \infty} A_{n}^{(2)} P(x, x) d \mu(x) \\
& \leq \int \widetilde{P}(x, x) d \mu(x) .
\end{aligned}
$$


Thus, if this integral is finite then so is $\operatorname{tr} P$, and the trace formula (4.5) follows from Theorem 3.1. Conversely, if $P$ is not traceable then both sides of (4.5) are infinite.

Since $\widetilde{P}(x, y)$ agrees with $P(x, y)$ at all regular points of continuity, Theorem 4.3 extends two previous generalizations of Mercer's theorem for continuous kernels due to Duflo ([3], Théorème V.3.3.1) and Reed and Simon ([6], Lemma to Theorem XI.31.)

\section{REFERENCES}

[1] C. Brislawn, Kernels of trace class operators, Proc. Amer. Math. Soc., 104 (1988), 1181-1190.

[2] J. L. Doob, Stochastic Processes, John Wiley \& Sons, New York, 1953.

[3] M. Duflo, Généralités sur les représentations induites, Représentations des Groupes de Lie Résolubles, Monographies de la Soc. Math. de France, vol. 4, Dunod, Paris, (1972), 93-119.

[4] R. Edwards and G. Gaudry, Littlewood-Paley and Multiplier Theory, Ergebnisse Math. und Grenz., vol. 90, Springer-Verlag, Berlin, Heidelberg, New York, 1977.

[5] B. Muckenhoupt, Weighted norm inequalities for the Hardy maximal function, Trans. Amer. Math. Soc., 165 (1972), 207-226.

[6] M. Reed and B. Simon, Scattering Theory, Methods of Modern Mathematical Physics, vol. 3, Academic Press, New York, 1979.

[7] F. Riesz and B. Sz.-Nagy, Functional Analysis, Frederick Ungar Publishing Co., New York, 1955.

[8] E. M. Stein, Topics in Harmonic Analysis, Annals of Math. Studies, vol. 63, Princeton Univ. Press, Princeton, NJ, 1970.

[9] A. Torchinsky, Real-Variable Methods in Harmonic Analysis, Pure and Appl. Math., vol. 123, Academic Press, Orlando, FL, 1986.

[10] F. Tricomi, Integral Equations, Dover Publications, New York, 1985.

[11] J. Weidmann, Integraloperatoren der spurklasse, Math. Ann., 163 (1966), 340345 .

Received February 13, 1990 and in revised form April 27, 1990.

UNIVERSITY OF SOUTHERN CALIFORNIA

Los ANGELES, CA 90089-1113 


\section{PACIFIC JOURNAL OF MATHEMATICS EDITORS}

\author{
V. S. VARADARAJAN \\ (Managing Editor) \\ University of California \\ Los Angeles, CA 90024-1555-05 \\ Herbert Clemens \\ University of Utah \\ Salt Lake City, UT 84112 \\ THOMAS ENRIGHT \\ University of California, San Diego \\ La Jolla, CA 92093
}

R. FINN

Stanford University

Stanford, CA 94305

HeRmann FlaschKa

University of Arizona

Tucson, AZ 85721

VAUGHAN F. R. JoNES

University of California

Berkeley, CA 94720

SteVen KeRCKHOFF

Stanford University

Stanford, CA 94305
C. C. MOORE

University of California

Berkeley, CA 94720

MaRTin ScharLemanN

University of California

Santa Barbara, CA 93106

HAROLd STARK

University of California, San Diego

La Jolla, CA 92093

\section{ASSOCIATE EDITORS \\ R. ARENS \\ E. F. BECKENBACH (1906-1982) \\ B. H. NeumanN \\ F. WoLf \\ (1904-1989) \\ K. YoshidA \\ SUPPORTING INSTITUTIONS \\ UNIVERSITY OF ARIZONA \\ UNIVERSITY OF BRITISH COLUMBIA \\ CALIFORNIA INSTITUTE OF TECHNOLOGY \\ UNIVERSITY OF CALIFORNIA \\ MONTANA STATE UNIVERSITY \\ UNIVERSITY OF NEVADA, RENO \\ NEW MEXICO STATE UNIVERSITY OREGON STATE UNIVERSITY \\ UNIVERSITY OF OREGON \\ UNIVERSITY OF SOUTHERN CALIFORNIA \\ STANFORD UNIVERSITY \\ UNIVERSITY OF HAWAII \\ UNIVERSITY OF TOKYO \\ UNIVERSITY OF UTAH \\ WASHINGTON STATE UNIVERSITY UNIVERSITY OF WASHINGTON}

The Supporting Institutions listed above contribute to the cost of publication of this Journal, but they are not owners or publishers and have no responsibility for its content or policies.

Mathematical papers intended for publication in the Pacific Journal of Mathematics should be in typed form or offset-reproduced (not dittoed), double spaced with large margins. Please do not use built up fractions in the text of the manuscript. However, you may use them in the displayed equations. Underline Greek letters in red, German in green, and script in blue. The first paragraph must be capable of being used separately as a synopsis of the entire paper. In particular it should contain no bibliographic references. Please propose a heading for the odd numbered pages of less than 35 characters. Manuscripts, in triplicate, may be sent to any one of the editors. Please classify according to the 1991 Mathematics Subject Classification scheme which can be found in the December index volumes of Mathematical Reviews. Supply name and address of author to whom proofs should be sent. All other communications should be addressed to the managing editor, or Elaine Barth, University of California, Los Angeles, California 90024-1555-05.

There are page-charges associated with articles appearing in the Pacific Journal of Mathematics. These charges are expected to be paid by the author's University, Government Agency or Company. If the author or authors do not have access to such Institutional support these charges are waived. Single authors will receive 50 free reprints; joint authors will receive a total of 100 free reprints. Additional copies may be obtained at cost in multiples of 50 .

The Pacific Journal of Mathematics (ISSN 0030-8730) is published monthly except for July and August. Regular subscription rate: $\$ 190.00$ a year (10 issues). Special rate: $\$ 95.00$ a year to individual members of supporting institutions.

Subscriptions, orders for numbers issued in the last three calendar years, and changes of address should be sent to Pacific Journal of Mathematics, P.O. Box 969, Carmel Valley, CA 93924, U.S.A. Old back numbers obtainable from Kraus Periodicals Co., Route 100, Millwood, NY 10546.

The Pacific Journal of Mathematics at P.O. Box 969, Carmel Valley, CA 93924 (ISSN 0030-8730) is published monthly except for July and August. Second-class postage paid at Carmel Valley, California 93924, and additional mailing offices. Postmaster: send address changes to Pacific Journal of Mathematics, P.O. Box 969, Carmel Valley, CA 93924.

PUBLISHED BY PACIFIC JOURNAL OF MATHEMATICS, A NON-PROFIT CORPORATION Copyright (C) 1991 by Pacific Journal of Mathematics 


\section{Pacific Journal of Mathematics}

Vol. 150, No. $2 \quad$ October, 1991

Selman Akbulut and Henry Churchill King, Rational structures on

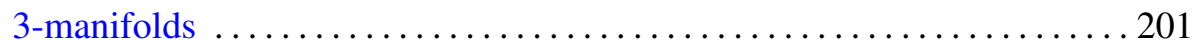

Mark Baker, On coverings of figure eight knot surgeries $\ldots \ldots \ldots \ldots \ldots 215$

Christopher Michael Brislawn, Traceable integral kernels on countably generated measure spaces . ...........................229

William Chin, Crossed products and generalized inner actions of Hopf algebras

Tadeusz Figiel, William Buhmann Johnson and Gideon Schechtman, Factorizations of natural embeddings of $l_{p}^{n}$ into $L_{r}$. II $\ldots \ldots \ldots \ldots \ldots 261$

David Howard Gluck, Character value estimates for groups of Lie type ... 279

Charn-Huen Kan, Norming vectors of linear operators between $L_{p}$

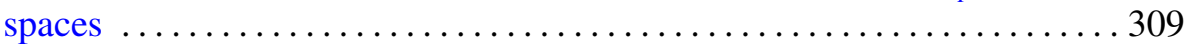

Marko Kranjc, Embedding a 2-complex $K$ in $\mathbb{R}^{4}$ when $H^{2}(K)$ is a cyclic

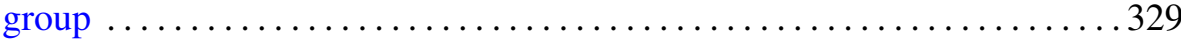

Ka-Lam Kueh, The remainder terms aspect of the theory of the Riemann

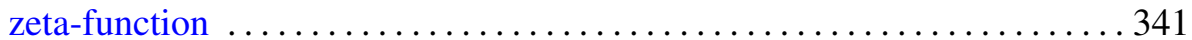

J. A. Marti, Sur la rigidité comparée de fonctions, distributions, ou hyperfonctions analytiques par rapport à un groupe de variables

Margherita Roggero and Paolo Valabrega, Chern classes and cohomology for rank 2 reflexive sheaves on $\mathbf{P}^{3}$ 